\title{
Switchable radical carbonylation by polarity-regulation
}

\author{
Bin $\mathrm{Lu}^{1}$, Xiaotian $\mathrm{Qi}^{2}$, Wen-Jing Xiao ${ }^{1, *}$, Jia-Rong Chen ${ }^{1, *}$
}

${ }^{1}$ Key Laboratory of Pesticides \& Chemical Biology Ministry of Education, College of Chemistry, Central China Normal University, 152 Luoyu Road, Wuhan, Hubei 430079, People’s Republic of China

${ }^{2}$ Engineering Research Center of Organosilicon Compounds \& Materials, Ministry of Education, College of Chemistry and Molecular Sciences, Wuhan University, Wuhan 430072, P. R. China, People's Republic of China

*wxiao@mail.ccnu.edu.cn; chenjiarong@mail.ccnu.edu.cn

Carbonylation reactions involving $\mathrm{CO}$ as readily available $\mathrm{C} 1$ synthons have become one of the most important tools for construction of carbonyl compounds from feedstock chemicals in modern chemical synthesis. Whereas numerous catalytic methods for carbonylation reactions proceeding via ionic or radical pathways have been reported, an inherent limitation to these methods is the need to control switchable single and double carbonylative formation of value-added products from the same and simple starting materials. Here we describe a new strategy that exploits simple visible-light-driven photoredox catalysis to regulate the polarity of coupling partners to drive switchable radical carbonylation reactions. Controlled trap of various alkyl radicals by single or double co thereby proceed smoothly with excellent selectivity in the presence of various amine nucleophiles at room temperature, generating valuable amides and $\alpha$-ketoamides in a versatile and controlled fashion. Combined experimental and DFT computational studies suggest that trap of the initially formed acyl radical by the second molecule of $\mathrm{CO}$ to form $\alpha$-ketoacyl radical is a facile but reversible process; and photoredox-catalyzed SET oxidation of natural nucleophilic amines into relatively electrophilic nitrogen radical cations is responsible for switchable coupling with such two radical intermediates.

Carbonylation reactions with carbon monoxide (CO) as the cheapest and most readily available $\mathrm{C} 1$ synthons have become one of the most important tools for construction of various significant carbonyl compounds both in industry and academia. By manipulating the steric or electronic features of the ligands, chemists over the past several decades have developed many efficient transition metal catalyst systems for CO-based carbonylation of aryl, vinyl, allyl, and benzyl halides (Fig. 1A). ${ }^{1-2}$ As for the challenging alkyl halides that typically encounter inherent slow oxidative addition to the metal and competitive $\beta$-hydride elimination of alkyl metal species resulting isomerization of alkyl metal species, ${ }^{3}$ radical carbonylation reactions provide a conceptually new and practical alternative way to carbonylate such compound class. ${ }^{4-6}$ Remarkably, Ryu developed a robust ultraviolet (UV) light-induced Pd-catalyzed atom transfer strategy for radical carbonylation reaction of alkyl iodides, significantly expanding the breadth and utility of the carbonylation chemistry over either the original radical carbonylation reactions or metal-catalyzed carbonylation reactions. ${ }^{7}$ Arndtsen recently documented that simple visible-light excitation of palladium-based catalyst enabled both oxidative addition and reductive elimination to proceed with low barriers. ${ }^{8,9}$ This novel activation strategy first allowed facile radical carbonylative conversion of both aryl and alkyl halides to acid chlorides that undergo versatile couplings with difficult nucleophiles under mild conditions. Polyzos disclosed a unique tandem photocatalytic cycle of $\left[\operatorname{Ir}(\mathrm{ppy})_{2}(\mathrm{dtb}-\mathrm{bpy})\right] \mathrm{PF}_{6}$ for 
carbonylative amidation of (hetero)aryl and alkyl halides using CO under continuous flow condition. ${ }^{10}$ Building on the special adsorbability of chitin-derived carbon nanofibrous microspheres (CNMs), Lei and Zhang reported a breakthrough method of employing CNMs as a promoter to realize an oxidative double radical carbonylation of alkanes for synthesis of various alkyl $\alpha$-ketoamides using stoichiometric di-tert-butyl peroxide (DTBP) as oxidant. ${ }^{11}$ In addition to these inspiring studies, numerous other catalytic methods for single or double carbonylation reactions proceeding via ionic or radical pathways have also been reported and extensively studied. ${ }^{12-16}$ However, to our knowledge, a generally applicable catalytic system that allowed controlled switchable single and double carbonylative formation of value-added products from the same and simple starting materials is still unknown. ${ }^{17-19}$

Transition metal-catalyzed mono and double carbonylation reactions are strictly dependent on many factors such reaction conditions (CO pressure, temperature, steric or electronic features of catalysts), substrates, types of nucleophiles. ${ }^{17}$ Considering its inherent two-electron activation mechanism, the factors that favor activation of substrates via oxidative addition ultimately inhibit subsequent carbonylative bond-forming reductive elimination. Moreover, strong coordinating ability and reducibility of $\mathrm{CO}$ often influence the activity of metal catalyst and thus increased temperature and high CO pressure are usually required. As a result, it is a great challenge to achieve ideal mono- and double carbonylation selectivity by simple fine tuning the reaction conditions in transition metal-catalyzed processes. Despite being a potentially practical method particularly in the context of carbonylation of alkyl halides, radical-mediated carbonylation methods towards switchable mono and double carbonylation also appear unlikely to provide a viable alternative. For instance, no evidence for sequential trap of radicals by two molecules of CO was observed in Coffman's radical copolymerization of ethylene and CO even at extremely high CO pressures (>1000 atm, ethylene/CO = 3/7) ${ }^{20,21}$ This study suggested that further trap of an acyl radical by $\mathrm{CO}$ occurs with great difficulty. ${ }^{22,23}$ In the study of UV light/Pd-promoted atom transfer radical carbonylation of alkyl halides, Ryu reported that in some cases a mixture of singly and double carbonylated products, amides and $\alpha$-ketoamides, could be obtained but with low selectivity (Fig. 1B). ${ }^{4,24-26}$ The moderate mono/double carbonylation ratios could be attributed to the simultaneous involvement of acylpalladium and acylcarbamoylpalladium complexes in this process, suggesting that it is challenging to improve by simply modification of catalytic systems. Recently, it has become recognized that the philicity of simple organic substrates could be altered by conversion to various open-shell radical species upon visible-light-driven photoredox-catalyzed single-electron transfer (SET), and that these changes can be exploited to design otherwise polarity-mismatched transformations (Fig. 1C).27-28 Considering the radical properties of acyl and $\alpha$-ketoacyl radicals, we hypothesized that photoredox catalysis-mediated regulation of the polarity of nucleophilic coupling partners would provide a conceptually new alternative strategy for switchable radical carbonylation reactions. For example, visible-light irradiation of several families of amine and derivatives is known to promote efficient formation of various electrophilic, nucleophilic or ambiphilic nitrogen radical species under SET reduction or oxidation conditions (Fig. 1C). ${ }^{29-32}$ Thus, we questioned whether the merger of photoredox redox-mediated nitrogen radical chemistry with radical carbonylation could provide a generally applicable method for controlled mono and double radical carbonylative amidation reactions.

Despite the extensive kinetic studies on single radical carbonylation, the double radical carbonylation is largely unexplored, and to the best of our knowledge, there is still even no kinetic information about the further trap of acyl radicals and the reverse process. ${ }^{4}$ Therefore, DFT calculations were initially carried out to investigate the trap of model intermediate $n$-butyl acyl radical I by another molecule of CO (Fig. 1D). Computational results show that the formation of $\alpha$-ketoacyl radical II through the trap of acyl radical I by 
CO only requires an energy barrier of $7.2 \mathrm{kcal} \mathrm{mol}^{-1}$. Moreover, the calculated reaction energy $\left(\Delta G_{\mathrm{rxn}}\right)$ indicates that these two radical species have comparable stability, and the transformation between radicals II and $\mathbf{I}$ is predicted to be kinetically feasible but a reversible process. Moreover, the calculated Global nucleophilicity and electrophilicity suggest that $\mathrm{n}$-butyl acyl radical I has a stronger nucleophilicity while $\alpha$-ketoacyl radical II has a stronger electrophilicity. ${ }^{33-35}$ Collectively, these observations support the anticipated interplay of polar effects and show promise for implementation in switchable radical carbonylation. Specifically, though the addition of acyl radical to $\mathrm{CO}$ is only slightly exothermic, polarity-match between $\alpha$-keto acyl radical and natural nucleophilic amine would probably provide the thermodynamic driving force to this process. In contrast, relatively more nucleophilic acyl radical should be more easily intercepted by electrophilic aminium radical.

Here, we describe the successful realization of the concept of polarity-regulation and its implementation as part of a mild and general method for switchable CO-based mono and double carbonylation of diverse easily accessible alkyl radicals with excellent selectivity in the presence of various amines at room temperature (Fig. 1E). This protocol is mild, robust, selective, and most importantly, capable of tolerating a wide range of radical precursors and functional groups, providing a practical and efficient method for synthesis of valuable $\alpha$-ketoamides and amides from the same starting materials in a controlled and versatile fashion. ${ }^{36,37}$

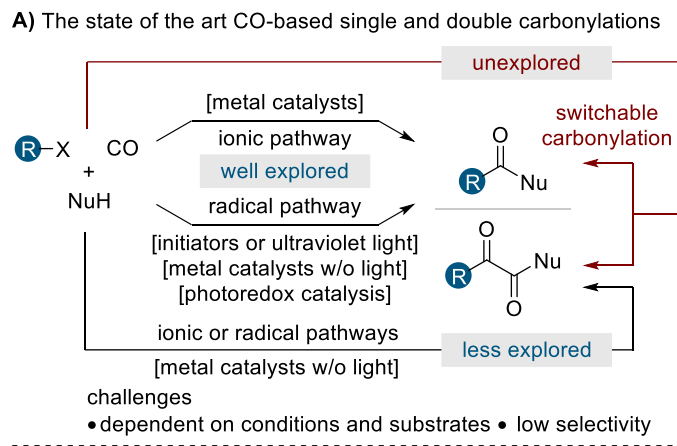

D) Theoretical study on the reactivity of acyl and $\alpha$-ketoacyl radicals

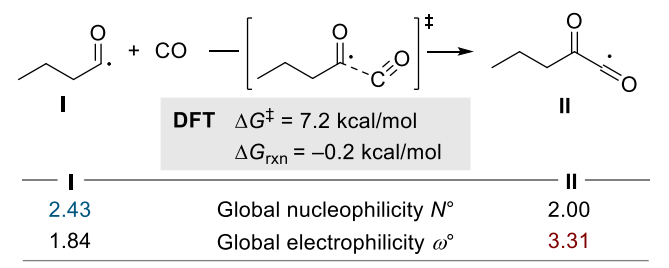

B) Ryu's Pd/UV light system

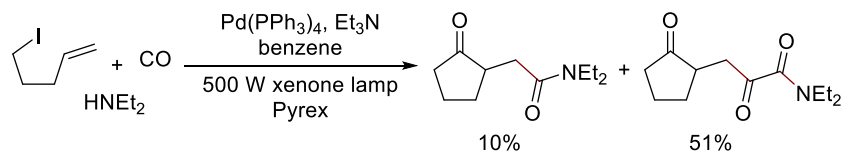

C) Our concept: polarity-regulation strategy

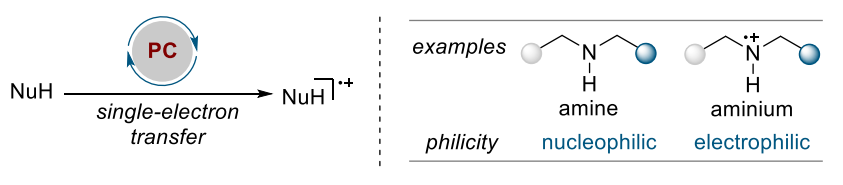

E) This work: controlled switchable radical carbonylation

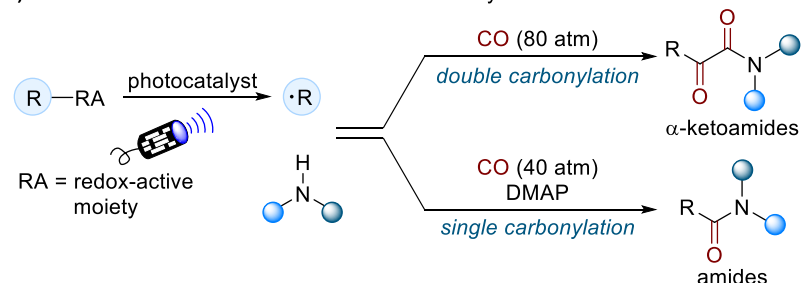

- readily available substrates and broad scope

- redox-neutral, simple, and mild reaction conditions

- excellent selectivity for double and single carbonylation

- switchable access to valuable $\alpha$-ketoamides and amides

Fig. 1. Background and evolution of a strategy for switchable radical carbonylation. (A) Overview of the main methods for CO-based carbonyl reactions. (B) Pioneering radical carbonylation by Pd/UV light system. (C) Development of a polarity-regulation strategy by single electron-transfer (SET). Conversion of natural nucleophilic amine to electrophilic aminium. PC = photoredox catalysis. (D) Computational studies on the trap of $\mathrm{n}$-butyl acyl radical by $\mathrm{CO}$ and reactivities of acyl radical and $\alpha$-ketoacyl radical. DFT, density functional theory; $\Delta G^{\ddagger}=$ activation free energy; $\Delta G_{r x n}=$ Gibbs reaction free energy. (E) Application of polar-regulation strategy in controlled switchable single and double radical carbonylation reactions with $\mathrm{CO}$ as $\mathrm{C} 1$ building block and amines as coupling partners. DMF, N,N-Dimethylformamide; DMAP, 4-dimethylaminopyridine. 


\section{Reaction development}

Oxime esters that could be easily prepared from commercial ketones and carboxylic acids have recently proved to be a class of versatile cyanoalkyl radical precursors for many radical cross-coupling reactions under photoreduction conditions, as shown by us $^{38}$ and other groups. ${ }^{39}$ To explore the application of polarity-regulation strategy in switchable radical carbonylation reactions, we chose a three-component carbonylative coupling among oxime ester $\mathbf{1} \mathbf{a}$, aniline $\mathbf{2} \mathbf{a}$, and $\mathrm{CO}(80 \mathrm{~atm})$ as a model reaction (Fig. 2A). The experiment was performed in a stainless-steel autoclave equipped with two quartz glass windows that served as a pressure-resistant apparatus under visible-light irradiation ( $2 \times 3 \mathrm{~W}$ blue LEDs, Fig. S6). The major challenge was to control chemoselectivity (single vs double carbonylation), while direct two-component coupling of $1 \mathbf{a}$ and $\mathbf{2 a}$ might also be a competing a side reaction. At the outset, we were particularly interested to test whether the double carbonylation could work using commonly used $f a c-\operatorname{Ir}(\mathrm{ppy})_{3}$ as photocatalyst. After extensive screening of the various reaction parameters, we found that the desired double carbonylation indeed worked smoothly, but highly dependent on reaction conditions. Notably, both the reaction efficiency and single/double carbonylation product ratio appeared to be very sensitive to the media (Fig. 2B). For example, the reaction performed in DMF at room temperature proceed smoothly to give the double carbonylation product, $\alpha$-ketoamide 3aa in $74 \%$ yield, together with single carbonylation product amide 4 aa in $2 \%$ yield. By contrary, the use of $\mathrm{CH}_{3} \mathrm{CN}$ gave comparable yield of 3aa, but a significant amount of 4 aa was observed ( $18 \%$ yield) while the reason is still unclear. Interestingly, in the case of DMSO, amide 4aa was formed as the major product accompanied by a $12 \%$ yield of 3aa. Other solvents such as toluene, $\mathrm{CH}_{2} \mathrm{Cl}_{2}$, and THF led to an obvious decrease in yields of both 3aa and 4aa as well as their ratios. Other commonly used Ir- and Ru-based photocatalysts, including $\left[\operatorname{Ir}(\mathrm{ppy})_{2}(\mathrm{dtbbpy})\right] \mathrm{PF}_{6},\left[\operatorname{Ir}\left(\mathrm{dF}\left(\mathrm{CF}_{3}\right) \mathrm{ppy}_{2}(\mathrm{dtbbpy})\right] \mathrm{PF}_{6},\left[\mathrm{Ru}(\mathrm{bpy})_{3}\right] \mathrm{Cl}_{2} \cdot 6 \mathrm{H}_{2} \mathrm{O}\right.$, and $\mathrm{Ru}(\mathrm{bpz})_{3}\left(\mathrm{PF}_{6}\right)_{2}$, all resulted in lower yields of 3aa (47-61\%) or poor 3aa/4aa selectivity. Moreover, lower pressure of CO resulted in a significant decrease in conversion to both 3aa and 4aa mainly due to the difficulty in trapping of cyanoalkyl radical by $\mathrm{CO}$.

Encouraged by the formation of variable amounts of single carbonylation product amide 4 aa in the double carbonylation processes, we proceed to identify suitable conditions to improve the selectivity towards 4aa. Our preliminary DFT calculation studies on the global nucleophilicity and electrophilicity of model $n$-butyl radical-derived acyl radical and $\alpha$-keto acyl radical demonstrated that the acyl radical has a relatively stronger nucleophilicity compared to $\alpha$-ketoacyl radical (Fig. 1D). According to our polarity-regulation strategy (Fig. 1C), if the natural nucleophilic aniline $\mathbf{2 a}$ could be firstly transformed to electrophilic aminium radical by SET oxidation, such a species would probably more easily couple with acyl radical to generate amide 4 aa as the main product. In recent years, the ability of proton-coupled electron transfer (PCET) to activate strong $\mathrm{N}-\mathrm{H}$ bonds to access various nitrogen radicals have been extensively exploited in photoredox-catalyzed C-N bond-forming reactions. ${ }^{40,41}$ Many redox or simple electron-transfer steps that are estimated to be endergonic by simple comparison of redox potentials become exergonic, when the electron transfer is coupled with proton transfer. Thus, we attempted to add base to facilitate the SET oxidation of aniline $\mathbf{2} \mathbf{a}$ into the corresponding aminium radical. We were pleased to discover that the addition of stoichiometric 4-dimethylaminopyridine (DMAP) (3.0 equiv.) could completely reverse the product distribution to the predominant formation of single carbonylation product 4aa under otherwise same conditions ( $80 \%$ GC yield, Fig. 2C). Further simple evaluation of pyridine derivatives A1-A7 disclosed that the electronic property of substituents at the para-position of pyridine ring appeared to be critical to both reaction efficiency and product distribution. Electron-donating groups were typically superior to electron-withdrawing ones. Surprisingly, in the case of $\mathrm{N}, \mathrm{N}$-dimethylaniline, 
neither 3aa nor 4aa was formed, implying that the pyridine ring was indispensable. Notably, the desired single carbonylation also proceeded smoothly even under $40 \mathrm{~atm}$ of $\mathrm{CO}$ in the presence of DMAP, giving 4aa in $83 \%$ yield. In addition to pyridine derivatives, a range of other commonly used bases including inorganic and organic ones showed similar effect, with 4aa being preferentially obtained as main product but with decreased yields (25-68\%), together with variable amounts of 3aa (Table S7). These results suggested that DMAP should work as a base to engage in PCET-mediated SET oxidation of aniline 2a under photoredox catalytic conditions. Finally, a range of control experiments also established that double and single carbonylation reactions are photochemical processes, and each parameter is critical to both transformations.

A)

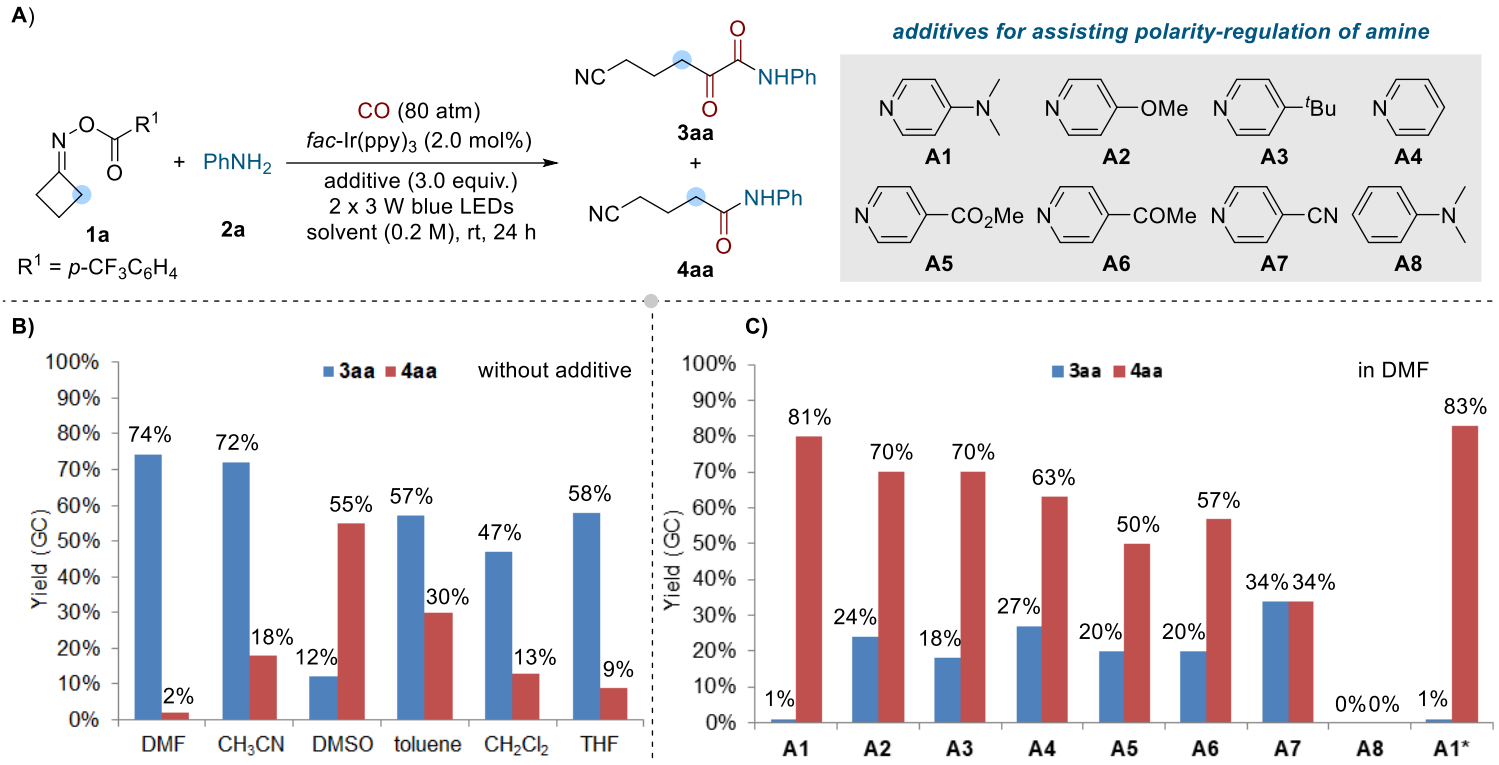

Fig. 2. Development of controlled switchable radical carbonylation and reaction optimization. (A) Switchable radical carbonylation of oxime ester $\mathbf{1 a}$, aniline 2a, and CO. (B) Effect of the solvents on the reaction efficiency and single carbonylation selectivity. All yields are GC yields. (C) Effect of additives on double radical carbonylation by polarity-regulation of amine. All yields are GC yields. *With $40 \mathrm{~atm}$ of CO.

\section{Reaction scope}

With two sets of optimized conditions in hand, we investigated the reaction scope by reacting a wide variety of commercially available amines $\mathbf{2}$ with oxime ester 1a (Fig. 3). First, we examined a representative set of primary aryl and heteroaryl amines, and found that the scope proved to quite general with respect to both the steric and electronic properties (Fig. 3A). For example, aniline $2 \mathrm{a}$ and its analogues $\mathbf{2 b}$-2j with various electron-donating (e.g., $\mathrm{Me}, \mathrm{Et}, \mathrm{OMe}$ ) or electron-withdrawing (e.g., $\mathrm{CF}_{3}, \mathrm{~F}$, $\mathrm{Cl}, \mathrm{Br}$ ) functional groups at the ortho-, meta- or para-position of the phenyl ring could all participate in desired double and mono radical carbonylation reactions smoothly. The corresponidng $\alpha$-ketoamides 3aa-3aj (51-73\%) and amides 4aa-4aj (46-86\%) in modest to good yields with excellent chemoselectivity. Moreover, both double and mono carbonylation ractions of 3,5-disubstituted aniline $\mathbf{2 k}$ and very sterically demanding aniline $\mathbf{2 l}$ also worked well, affording the desired products in satisfactory yields. The current divergent catalytic systems could be successfully extended to heteroaryl amine $\mathbf{2 m}$ and $\mathbf{N}$-Me-aniline $\mathbf{2 n}$ as well, leading to good-yield formation of products 3am-3an and 4am-4an, respectively. Markedly, the reactions were also found to be very general regarding the diversely substituted alkyl amines used. For 
instance, sterically hindered primary aliphatic amine $\mathbf{2 0}$ and acyclic secondary aliphatic amine $\mathbf{2 p}$ were well tolerated, delivering the expected $\alpha$-ketoamides and amides with useful yields. Note that reactions with various amine heterocycles, including piperidine (2q), piperazine (2r), morpholine (2s), and thiomorpholine (2t), also worked efficiently to produce target $\alpha$-keoamides 3aq-3at and amides 4aq-4at with good yields. The reactions of azepane $\mathbf{2 u}$ gave $3 a u$ and $\mathbf{4 a u}$ in $85 \%$ and $82 \%$ yields, respectively.

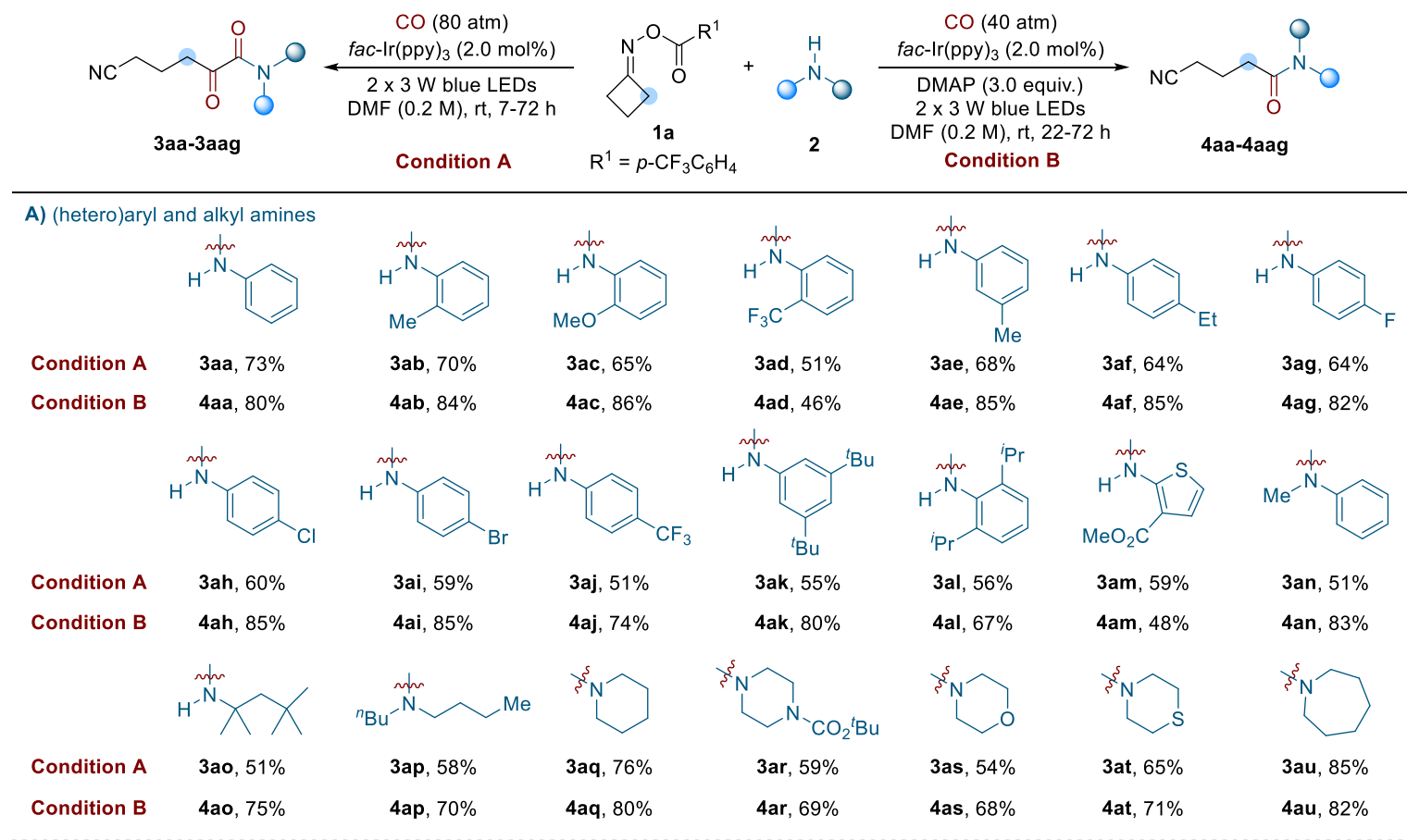

B) amine-containing drugs, drug fragments and amino acid derivative

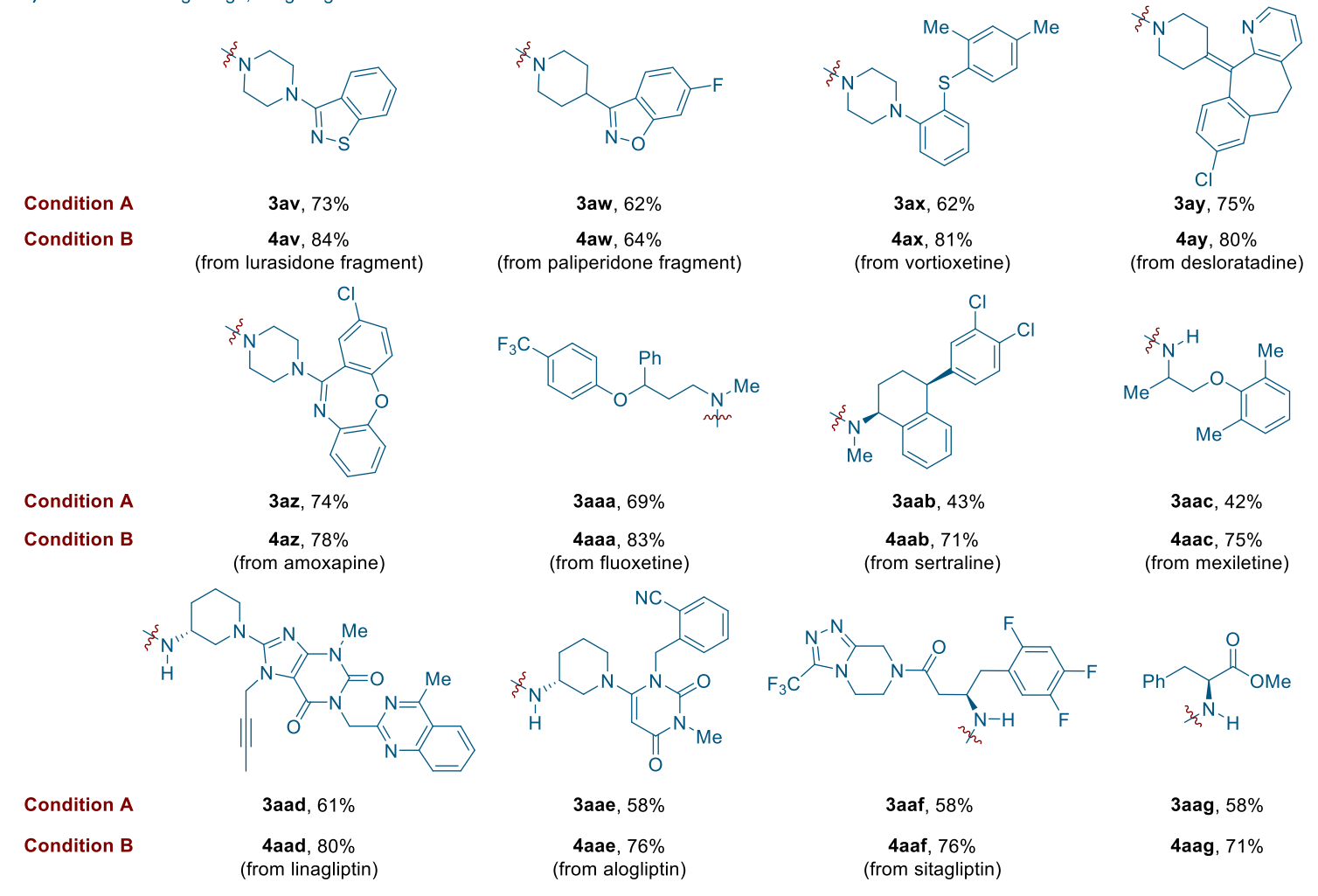

Fig. 3. Scope of amines in controlled switchable radical carbonylation reactions. Isolated yields for 
$\alpha$-ketoamides and amides after chromatography are given. (A) Scope of aryl, heteroaryl, and alkyl amines. (B) Application of the switchable radical carbonylation methodology in late-stage modification of biologically relevant molecules. ppy, 2-phenylpyridyl; DMF, $N, N$-Dimethylformamide; Me, methyl; $t$-Bu, tert-butyl; Ph, phenyl.

The primary and secondary amine functionalities widely exist in numerous pharmaceuticals and biologically relevant molecules. Considering the excellent functional-group tolerance of the current switchable and biological importance of amide and $\alpha$-ketoamide scaffolds, we next explored the method's further synthetic potential with respect to modifiying amine-containing, more structrually complex drug fragments, drugs, and amino acid derivative (Fig. 3B). Pleasingly, two drug fragments ( $2 \mathbf{v}$ and $\mathbf{2 w}$ ) and nine marked drugs containg secondary and primary amine moieties (2x-2af) all underwent controlled double and mono radical carbonylation reactions smoothly to furnish the desired products 3ax-3aaf and 4ax-4aaf with typically good yields. The facile and sucessful incorporation of cyanoalkyl and carbonyl functional groups into these commonly encountered pharmaceutically privileged architectures would aid the construction of new drug candidates. ${ }^{42}$ Notably, chiral amino acid derivative, (S)-methyl 2-amino-3-phenylpropanoate $\mathbf{2 a g}$, also proved to be a suitable substrate, with 3aag and 4aag being formed in good yields without obvious erosion of the enantiopurity.

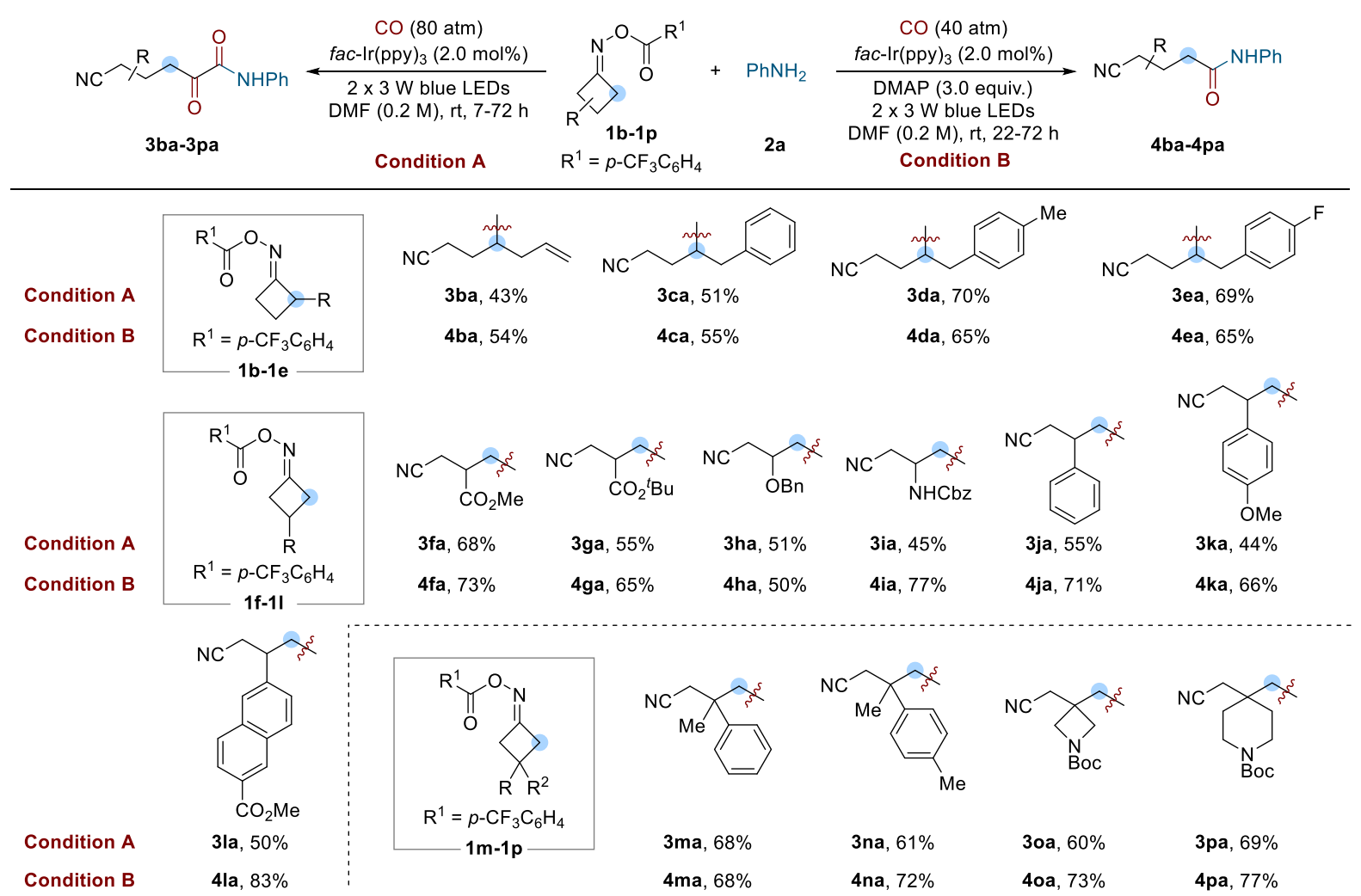

Fig. 4. Scope of oxime esters in switchable radical carbonylation reactions. Isolated yields fora-ketoamides and amides after chromatography are given. Me, methyl; $\mathrm{Bn}$, benzyl; $\mathrm{Cbz}$, benzyloxycarbonyl; Boc, tert-butoxycarbonyl.

Next, we turned our attention to explore the generality of the switchable radical carbonylation by reacting a selection of cyclobutanone oxime esters with aniline $\mathbf{2 a}$ under two sets of standard conditions (Fig. 4). We were pleased to find that structural variation of oxime moiety has no detrimental effects on the efficiency of reaction and chemoselectivity. For example, in addition to 1a, a range of oxime esters 
1b-1e having allyl, neutral, relatively electron-rich and electron-deficient benzyl groups at the 2-position reacted well to afford the corresponding doubly carbonylated $\alpha$-ketoamides 3ba-3ea and single carbonylated amides 4ba-4ea with generally good yields. Moreover, a variety of symmetric 3-monosubstituted oxime esters $\mathbf{1 f - 1 I}$ containing various functionalities such as ester, ether, Cbz-protected amine, and aryl were all tolerated. Both $\alpha$-ketoamides 3fa-3la and amides 4fa-4la were isolated in moderate to good yields. Interestingly, in most of these cases, double carbonylation reactions showcased somewhat higher yields. Remarkably, the reactions of 3,3-disubstituted oxime esters $1 \mathbf{m}$-1p proceeded smoothly, furnishing the corresponding $\alpha$-ketoamides 3ma-3pa and amides 4ma-4pa with comparable yields. Collectively, these results demonstrated that our divergent photocatalytic systems were suitable for diversely functionalized primary and secondary cyanoalkyl radicals.

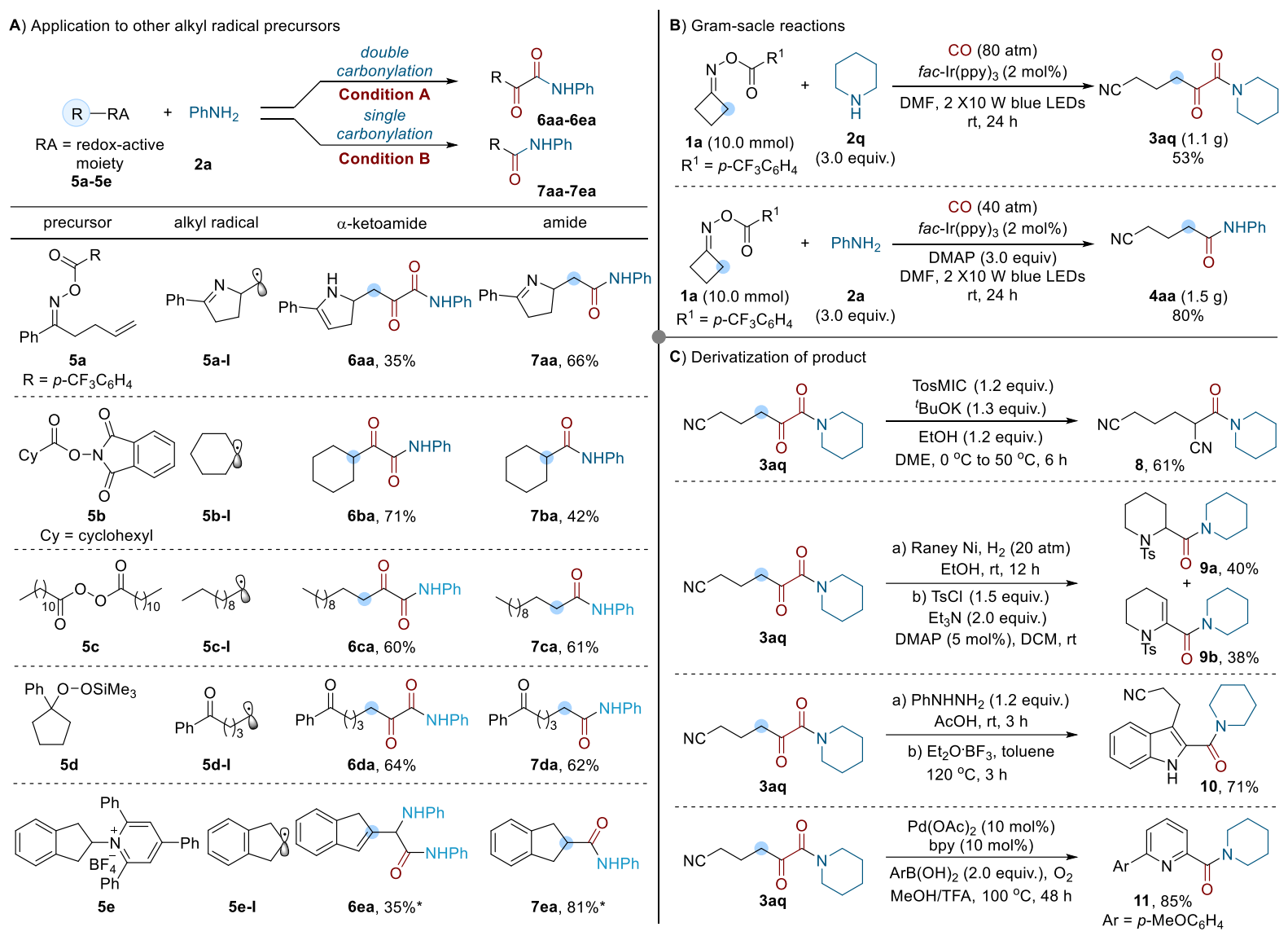

Fig. 5. Application to other types of radical precursors and synthetic applications. Isolated yields for $\alpha$-ketoamides and amides after chromatography are given. (A) Application to other types of alkyl radical precursors. (B) Scaled-up version of the switchable radical carbonylation reaction. (C) Derivatization of product $\alpha$-ketoamide. bpy, 2,2'-bipyridyl. *Using $\operatorname{Ir}(4-\mathrm{Fppy})_{3}$ as photocatalyst.

\section{Synthetic applications}

Having evaluated the scope of both reaction components, we sought to further show the utility of the switchable radical carbonylation protocol towards other radical precursors, and discovered that substrate scope can be successfully extended far beyond oxime esters (Fig. 5A). Our preliminary results illustrated that a representative range of redox-active substrates could also readily participate in the desired switchable radical carbonylation reactions under photoredox catalyzed conditions. For example, $\gamma$-alkenyl $\mathbf{O}$-acyl oxime 5a, which could undergo photoredox-catalyzed SET-reduction to generate iminyl radical 
followed by subsequent intramolecular 5 -exo-cyclization to form carbon radical $5 \mathrm{a}-\mathrm{I},{ }^{43}$ proved to be a suitable substrate. Thus, in the presence aniline $\mathbf{2 a}$, selective double and single carbonylations of radical intermediate $5-1$ occurred to furnish pyrroline-containing $\alpha$-ketoamide 6 aa and amide 7 aa in moderate to good yields. In addition, aliphatic carboxylic acid-derived $\mathrm{N}$-(acyloxy)-phthalimide $\mathbf{5 b}$ and alkyl diacyl peroxide $\mathbf{5 c}$ both could also serve as competent secondary and primary alkyl radical precursors to take part in divergent double and single radical carbonylation reactions smoothly. And the desired products were obtained with synthetically useful yields. Notably, the reaction of alkylsilyl peroxide $\mathbf{5} \mathbf{d}$, which was a good source of alkyl radical $\mathbf{5 d - I}$ upon SET-reduction-mediated ring-opening, ${ }^{44}$ proceeded well under the two sets of optimized conditions, leading to remote double and mono carbonylation products $6 \mathrm{da}$ and 7da with $64 \%$ and $62 \%$ yields, respectively. Primary alkyl amines represent a feedstock with a similar natural abundance as carboxylic acids. Alkyl amine-derived $\mathrm{N}$-alkyl pyridinium salt $\mathbf{5 e}$ that was commonly used as alkyl radical precursor, ${ }^{45}$ can also undergo switchable double and single carbonylation reactions via intermediate $\mathbf{5 e - I}$ to afford $\mathbf{6 e a}$ and $\mathbf{7 e a}$, respectively. On the basis of these results, we are convinced that the polarity-regulation-enabled switchable radical carbonylation approach will be extended to other photogenerated alkyl radicals. ${ }^{46}$

Notably, the current switchable radical carbonylation reactions could also be easily run on a gram scale. As exemplified by $\mathbf{3 a q}$ and $\mathbf{4 a a}$, two $10.0 \mathrm{mmol}$-scale reactions of $\mathbf{1 a}$ with piperidine $\mathbf{2 q}$ and aniline $2 \mathrm{a}$ as amine coupling partners proceeded well to afford $53 \%$ and $80 \%$ yields, respectively (Fig. 5B). Compared to the reactions performed at $0.2 \mathrm{mmol}$ scale, there was no significant loss on yields. Moreover, the versatile cyano and $\alpha$-ketoamide moieties present in the products provide many opportunities for synthetic manipulation (Fig. 5C). For instance, the $\alpha$-ketone moiety of 3aq was converted efficiently, in a single step, to cyano group by use of tosylmethyl isocyanide (TosMIC) in the presence of $t$-BuOK as a base, thus leading to dinitrile $\mathbf{8}$ in good yield. ${ }^{47}$ Moreover, when treated sequentially with Raney-Ni under a $\mathrm{H}_{2}$ atmosphere and tosyl chloride ( $\mathrm{TsCl}$ ), $\alpha$-ketoamide 3 aq could undergo intramolecular reductive amination to give Ts-protected piperidine derivatives $9 a$ and $\mathbf{9 b}$ respectively with moderate isolated yields. ${ }^{48}$ Notably, by combining acid-promoted condensation with $\mathrm{N}$-phenyl hydrazine and Lewis acid-mediated Fischer indolization process in a one-pot manner, 3aq was easily converted into 2,3-disubstituted indole 10 in $71 \%$ yield. ${ }^{49}$ Interestingly, Pd-catalyzed cascade reaction of 3aq with arylboronic acid enabled synthesis of valuable 2,6-disubstituted pyridine $\mathbf{1 1}$ in $85 \%$ yield..$^{50}$

\section{Mechanistic studies}

To shed light on the mechanism, a series of mechanistic experiments and computational studies were conducted (Fig. 6). When stoichiometric radical scavengers, 2,2,6,6-tetramethyl-1-piperidinyloxy (TEMPO) and 1,1-diphenylethene, were added to the model reaction of $\mathbf{1 a}$ and $\mathbf{2 a}$ under the standard double carbonylation conditions, the formation of $\alpha$-ketoamide 3aa was completely suppressed (Fig. 6A). Instead, in these reactions, trapping adducts $\mathbf{1 2}$ and $\mathbf{1 3}$ could be isolated or detected respectively, confirming the radical property of this process and possible involvement of cyanoalkyl radical 1a-B and acyl radical 1a-C. Moreover, when acyclic oxime ester $\mathbf{1 4}$, which was often used as precursor to acyl radical 14-A, was subjected to double and single carbonylation conditions in the presence or absence of $\mathrm{CO}, \alpha$-ketoamide 15a and amide 15b were isolated in 54\% and 30\% yields, respectively (Fig. 6B). These results provide further evidence of a radical process and acyl radical involved in both double and single radical carbonylation reactions. Note that, in agreement with our optimization studies, only trace of product $\mathbf{1 5 b}$ was observed either without addition of DMAP, suggesting that DMAP was also critical to the coupling between acyl radical $\mathbf{1 4 - A}$ and aniline $\mathbf{2 a}$. In order to get deeper insight into the role of DMAP, oxidation 
potentials of aniline $\mathbf{2 a}$, DMAP, and their mixture were tested by using of cyclic voltammetry (Fig. S7). Though the measured redox potentials of $2 \mathrm{a}\left(E_{\mathrm{ox}}=+0.93 \mathrm{~V}\right.$ vs SCE$)$ and DMAP $\left(E_{o x}=+0.84 \mathrm{~V}\right.$ vs SCE) are

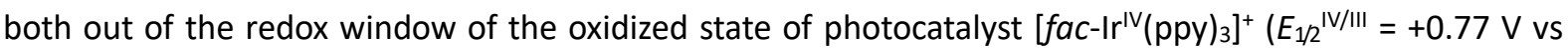
SCE), the redox potential of the mixture of $2 a$ and DMAP $\left(E_{\mathrm{ox}}=+0.72 \mathrm{~V}\right.$ vs $\left.S C E\right)$ is inside its window. Specifically, these results confirmed that SET-oxidation of $2 a$ became thermodynamically likely in the presence of DMAP.

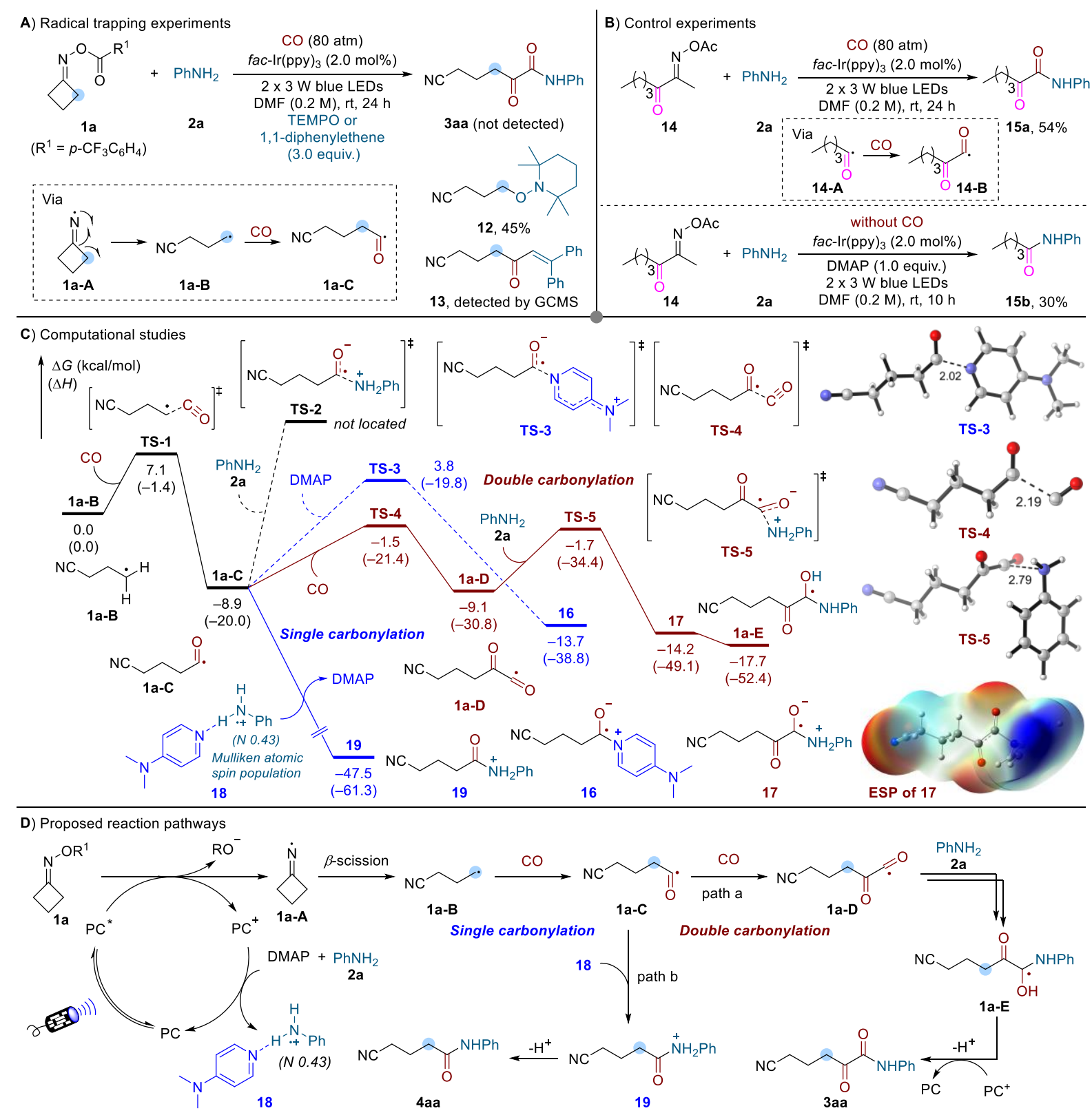

Fig. 6. Mechanistic, computational studies and plausible mechanisms. (A) Radical trapping experiments. (B) Control experiments of acyl radical precursor. (C) Free energy profiles for the divergent transformation pathways of cyanoalkyl radical 1a-B calculated at B3LYP/6-311+G(d,p)/SMD(DMF) level of theory. ESP = electrostatic potential map. (D) Proposed reaction pathways for the controlled mono and double radical carbonylation reactions.

Next, to gain a better understanding of the origin of chemoselective in the radical carbonylation reaction, some elementary steps starting from caynoalkyl radical 1a-B were evaluated through density 
functional theory (DFT) calculations at $B 3 L Y P / 6-311+G(d, p) / S M D(D M F)$ level of theory (Fig. $6 C$ ). The addition of cyanoalkyl radical 1a-B to CO through transition state TS-1 leads to the formation of acyl radical $\mathbf{1 a}-\mathrm{C}$, which is exergonic by $8.9 \mathrm{kcal} / \mathrm{mol}$. Subsequently, the acyl radical $\mathbf{1 a}-\mathbf{C}$ may encounter several competitive reaction pathways, including the radical addition to another molecule of CO via TS-4 and the direct nucleophilic addition of aniline $\mathbf{2} \mathbf{a}$ to $\mathbf{1} \mathbf{a}-\mathbf{C}$ via TS-2. Unlike the previously studied nucleophilic addition of aniline to benzoyl radical, ${ }^{51,52}$ we did not locate the structures of transition state TS-2 or the corresponding intermediates. We surmise that the lower stability of the intermediate is responsible for this outcome. On the other hand, the double carbonylation pathway through the radical addition transition state TS-4 $\left(\Delta G^{\ddagger}=7.4 \mathrm{kcal} / \mathrm{mol}\right)$ followed by the nucleophilic addition of aniline $2 \mathrm{a}$ to $\alpha$-ketoacyl radical 1a-D via TS-5 $\left(\Delta G^{\ddagger}=7.4 \mathrm{kcal} / \mathrm{mol}\right)$ is shown to be kinetically and thermodynamically feasible. The electrostatic potential map (ESP) of generated radical intermediate $\mathbf{1 7}$ shows that the negative and positive charge is located on the oxygen and nitrogen atoms, which reflects the dipolar character of $\mathbf{1 7 .}$ Thus, a facile intramolecular proton transfer may occur to form the hydroxymethyl radical 1a-E exothermically $(3.5 \mathrm{kcal} / \mathrm{mol})$. Sequential SET-oxidation of the $1 \mathrm{a}-\mathrm{E}$ by the oxidized photocatalyst and deprotonation would furnish the $\alpha$-ketoamide product 3 aa and regenerate the ground state photocatalyst.

Since highly selective single carbonylation reaction occurred only in the presence of DMAP, we envision that cyanoacyl radical $\mathbf{1 a}-\mathrm{C}$ may be intercepted by DMAP before reacting with the second molecule of $\mathrm{CO}$. As illustrated by the blue pathways in Fig. 6C, we studied the nucleophilic addition of DMAP to the cyanoacyl radical $\mathbf{1 a - C}$ via transition state TS-3. The higher energy barriers of transition state TS-3 indicates that this pathway cannot outcompete the radical addition transition state TS-4 in the double carbonylation pathway. Based on these negative results and our optimization studies, we realize that the hydrogen bonding interaction between DMAP and aniline $\mathbf{2 a}$ and ensuing SET-oxidation of $\mathbf{2 a}$ into radical species might be crucial for single radical carbonylation. Specifically, the formation of hydrogen bonding complex can enrich the electron density on aniline $\mathbf{2 a}$ part and thus promote the SET-oxidation of 2a to form an electrophilic radical cation $\mathbf{1 8}$ through a photoredox-catalyzed PCET process. By doing so, the natural nucleophilic aniline $\mathbf{2 a}$ was converted to relatively electrophilic aminium radical species $\mathbf{1 8}$, $^{53}$ leading to its preferential interception of nucleophilic cyanoalkyl 1a-C. Moreover, computational studies of the transformation of nitrogen radical cation 18 suggest that the Mulliken atomic spin population mainly locates at the nitrogen atom and its radical-radical cross-coupling with acyl radical $1 \mathrm{a}-\mathrm{C}$ is barrierless and highly exergonic, thus leading to intermediate 19 and final product amide 3aa after deprotonation. Taken together, in accordance with our design plan shown in Fig. 1C, DMAP-assisted polarity-regulation is the main reason for ensuring the controlled switchable radical carbonylation.

On the basis of these mechanistic studies, we proposed a plausible mechanism for this switchable radical carbonylation reaction with model substrates $\mathbf{1 a}$ and $\mathbf{2 a}$ (Fig. 6C). First, SET-reduction of redox-active oxime ester $\mathbf{1 a}$ by the excited state photocatalyst ${ }^{*} f a c-\operatorname{Ir}(\mathrm{ppy})_{3}$ produces iminyl radical $\mathbf{1 a}-\mathbf{A}$ and oxidizing state $f a c-\operatorname{Ir}^{\prime V}(\mathrm{ppy})_{3}{ }^{+}$with release of a carboxylic anion. Upon ring-opening $\beta-\mathrm{C}-\mathrm{C}$ bond cleavage, cyanoalkyl radical 1a-B could then be formed and intercepted by $\mathrm{CO}$ to generation cyanoalkylformyl radical 1a-C. At this stage, 1a-C would engage in two distinct pathways. Though our preliminary calculation studies show that the reaction of acyl radical with $\mathrm{CO}$ to form $\alpha$-ketoacyl radical is an equilibrium process and might be difficult to control due to the ready back reaction (Fig. 1D). In the absence of DMAP, the unique coupling of global electrophilic $\alpha$-ketoacyl radical with nucleophilic aniline 2a would drive the single-double-carbonylation equilibrium in the forward direction, thus furnishing hydroxymethyl radical 1a-E via intermediate 17. Finally, 1a-E undergoes SET-oxidation by the oxidizing 
state $f a c-\mid r^{1 V}(\mathrm{ppy})_{3}{ }^{+}$and deprotonation to afford $\alpha$-ketoamide 3aa, regenerating of the ground state photocatalyst fac-Ir(ppy $)_{3}$ and closing the photocatalytic cycle. In contrast, in the presence of DMAP, aniline 2a would first undergo SET-oxidation by the oxidizing state $f a c-\mid r^{1 \mathrm{~V}}(\mathrm{ppyy})_{3}{ }^{+}$through a PCET event to form nitrogen radical cation 18 . Given the polarity-match principle, coupling between electrophilic aminium radical 18 with global nuleophilic cyanoacyl radical 1a-C could be favored to generate intermediate 19 and the final product amide 4aa after deprotonation. Therefore, the reactivity characteristics of amines and aminiums caused by polarity-regulation are crucial to ensure exquisite chemoselectivity during the current switchable radical carbonylation reactions. Notably, both double and single carbonylation reactions are redox-neutral without the need for any external oxidant or reductant.

The results presented here illustrated that polarity-regulation strategy can drive controlled switchable carbonylation reactions of a range of carbon radicals. We are convinced that the broad substrate scope, good functional group tolerance, and modularity of this method for polarity-regulation of coupling partners should be of great utility to chemists in both academia and industry. ${ }^{54}$ Considering that photochemistry provides an enabling tool for generation of various radical intermediates that are otherwise difficult to produce in a controlled manner under thermal conditions, many exciting applications of this concept to other related switchable radical carbonylations under visible-light photoredox regimes can be anticipated. ${ }^{55}$

\section{REFERENCES AND NOTES}

1. M. Beller \& X.-F. Wu, Transition Metal Catalyzed Carbonylation Reactions: Carbonylative Activation of C-X Bonds, Springer, Berlin, 2013.

2. Peng, J.-B., Geng, H.-Q. \& Wu, X.-F. The Chemistry of CO: Carbonylation. Chem 5, 526-552 (2019).

3. Wu, L. et al. Palladium-Catalyzed Carbonylative Transformation of $\mathrm{C}\left(\mathrm{sp}^{3}\right)-\mathrm{X}$ Bonds. ACS Catal. 4, 2977-2989 (2014).

4. Ryu, I. \& Sonoda, N. Free-Radical Carbonylations: Then and Now. Angew. Chem. Int. Ed. 35, 1050-1066 (1996).

5. Ryu, I. Radical carboxylations of iodoalkanes and saturated alcohols using carbon monoxide. Chem. Soc. Rev. 30, 16-25 (2001).

6. Zhao, S. \& Mankad, N. P. Metal-catalysed radical carbonylation reactions. Catal. Sci. Technol. 9, 3603-3613 (2019).

7. Sumino, S., Fusano, A., Fukuyama, T. \& Ryu, I. Carbonylation Reactions of Alkyl lodides through the Interplay of Carbon Radicals and Pd Catalysts. Acc. Chem. Res. 47, 1563-1574 (2014).

8. Torres, G. M., Liu, Y. \& Arndtsen, B. A. A dual light-driven palladium catalyst: Breaking the barriers in carbonylation reactions. Science 368, 318-323 (2020).

9. Kathe, P. \& Fleischer, I. Light expands a catalyst's repertoire. Science 368, 242-243 (2020).

10. Forni, J. A., Micic, N., Connell, T. U., Weragoda, G. \& Polyzos, A. Tandem Photoredox Catalysis: Enabling Carbonylative Amidation of Aryl and Alkylhalides. Angew. Chem. Int. Ed. 59, 18646-18654 (2020).

11. Lu, L. et al. Carbon Nanofibrous Microspheres Promote the Oxidative Double Carbonylation of Alkanes with CO. Chem 4, 2861-2871 (2018).

12. Li, W. et al. From anilines to isatins: oxidative palladium-catalyzed double carbonylation of $\mathrm{C}-\mathrm{H}$ bonds. Angew. Chem. Int. Ed. 54, 1893-1896 (2015).

13. Zhang, Y., Geng, H.-Q. \& Wu, X.-F. Palladium-Catalyzed Perfluoroalkylative Carbonylation of 
Unactivated Alkenes: Access to beta-Perfluoroalkyl Esters. Angew. Chem. Int. Ed. 60, 24292-24298 (2021).

14. Guo, W. et al. Metal-Free, Room-Temperature, Radical Alkoxycarbonylation of Aryldiazonium Salts through Visible-Light Photoredox Catalysis. Angew. Chem. Int. Ed. 54, 2265-2269 (2015).

15. Cartier, A. et al. Carbonylation of Alkyl Radicals Derived from Organosilicates through Visible-Light Photoredox Catalysis. Angew. Chem. Int. Ed. 58, 1789-1793 (2019).

16. Majek, M. \& Wangelin, A. J. Metal-Free Carbonylations by Photoredox Catalysis. Angew. Chem. Int. Ed. 54, 2270-2274 (2015).

17. Das, D. \& Bhanage, B. M. Double Carbonylation Reactions: Overview and Recent Advances. Adv. Synth. Catal. 362, 3022-3058 (2020).

18. Singh, J., Sharma, S. \& Sharma, A. Photocatalytic Carbonylation Strategies: A Recent Trend in Organic Synthesis. J. Org. Chem. 86, 24-48 (2021).

19. Peng, J.-B. \& Wu, X.-F. Ligand- and Solvent-Controlled Regio- and Chemodivergent Carbonylative Reactions. Angew. Chem. Int. Ed. 57, 1152-1160 (2018).

20. Coffman, D. D., Pinkney, P. S., Wall, F. T., Wood, W. H. \& Young, H. S. Compositional Relationships in the Copolymerization of Ethylene with Carbon Monoxide. J. Am. Chem. Soc. 74, 3391-3393 (1952).

21. Brubaker, M. M., Coffman, D. D. \& Hoehn, H. H. Synthesis and Characterization of Ethylene/Carbon Monoxide Copolymers, A New Class of Polyketones1. J. Am. Chem. Soc. 74, 1509-1515 (1952).

22. Ryu, I., Sonoda, N. \& Curran, D. P. Tandem Radical Reactions of Carbon Monoxide, Isonitriles, and Other Reagent Equivalents of the Geminal Radical Acceptor/Radical Precursor Synthon. Chem. Rev. 96, 177-194 (1996).

23. Ryu, I. et al. New Radical Cascade Reactions Incorporating Multiple One-Carbon Radical Synthons: A Versatile Synthetic Methodology for Vicinal Singly and Doubly Acylated Oxime Ethers. J. Am. Chem. Soc. 121, 12190-12191 (1999).

24. Ryu, I. et al. Cascade Radical Reactions Catalyzed by a Pd/Light System: Cyclizative Multiple Carbonylation of 4-Alkenyl lodides. J. Am. Chem. Soc. 124, 3812-3813 (2002).

25. Fukuyama, T., Nishitani, S., Inouye, T., Morimoto, K. \& Ryu, I. Effective acceleration of atom transfer carbonylation of alkyl iodides by metal complexes. Application to the synthesis of the hinokinin precursor and dihydrocapsaicin. Org. Lett. 8, 1383-1386 (2006).

26. Fusano, A. et al. Pd/light-accelerated atom-transfer carbonylation of alkyl iodides: applications in multicomponent coupling processes leading to functionalized carboxylic acid derivatives. Chem. Eur. J. 18, 9415-9422 (2012).

27. Margrey, K. A. \& Nicewicz, D. A. A General Approach to Catalytic Alkene Anti-Markovnikov Hydrofunctionalization Reactions via Acridinium Photoredox Catalysis. Acc. Chem. Res. 49, 1997-2006 (2016).

28. Ravelli, D., Protti, S. \& Fagnoni, M. Carbon-Carbon Bond Forming Reactions via Photogenerated Intermediates. Chem. Rev. 116, 9850-9913 (2016).

29. Davies, J., Morcillo, S. P., Douglas, J. J. \& Leonori, D. Hydroxylamine Derivatives as Nitrogen-Radical Precursors in Visible-Light Photochemistry. Chem. Eur. J. 24, 12154-12163 (2018).

30. Chen, J.-R., Hu, X.-Q., Lu, L.-Q. \& Xiao, W.-J. Visible Light Photoredox-Controlled Reactions of N-Radicals and Radical lons. Chem. Soc. Rev. 45, 2044-2056 (2016).

31. Pitre, S. P. \& Overman, L. E. Strategic Use of Visible-Light Photoredox Catalysis in Natural Product Synthesis. Chem. Rev. 121, 10.1021/acs.chemrev.1c00247 (2021).

32. Constantin, T. et al. Aminoalkyl radicals as halogen-atom transfer agents for activation of alkyl and 
aryl halides. Science 367, 1021-1026 (2020).

33. Domingo, L. R. \& Pérez, P. Global and local reactivity indices for electrophilic/nucleophilic free radicals. Org. Biomol. Chem. 11, 4350-4358 (2013).

34. Qi, X., Zhu, L., Bai, R. \& Lan, Y. Stabilization of Two Radicals with One Metal: A Stepwise Coupling Model for Copper-Catalyzed Radical-Radical Cross-Coupling. Sci. Rep. 7, 43579 (2017).

35. Liu, S., Qi, X., Bai, R. \& Lan, Y. Theoretical Study of Ni-Catalyzed C-N Radical-Radical Cross-Coupling. J. Org. Chem. 84, 3321-3327 (2019).

36. Pattabiraman, V. R. \& Bode, J. W. Rethinking amide bond synthesis. Nature 480, 471-479 (2011).

37. De Risi, C., Pollini, G. P. \& Zanirato, V. Recent Developments in General Methodologies for the Synthesis of alpha-Ketoamides. Chem. Rev. 116, 3241-3305 (2016).

38. Yu, X.-Y., Zhao, Q.-Q., Chen, J., Xiao, W.-J. \& Chen, J.-R. When light meets nitrogen-centered radicals: From reagents to catalysts. Acc. Chem. Res. 53, 1066-1083 (2020).

39. Xiao, F., Guo, Y. \& Zeng, Y.-F. Recent Developments in Radical Cross-Coupling of Redox-Active Cycloketone Oximes. Adv. Synth. Catal. 363, 120-143 (2021).

40. Gentry, E. C. \& Knowles, R. R. Synthetic Applications of Proton-Coupled Electron Transfer. Acc. Chem. Res. 49, 1546-1556 (2016).

41. Nguyen, L. Q. \& Knowles, R. R. Catalytic C-N Bond-Forming Reactions Enabled by Proton-Coupled Electron Transfer Activation of Amide N-H Bonds. ACS Catal. 6, 2894-2903 (2016).

42. Kumar, R., Floden, N. J., Whitehurst, W. G. \& Gaunt, M. J. A general carbonyl alkylative amination for tertiary amine synthesis. Nature 581, 415-420 (2020).

43. Shen, X., Huang, C., Yuan, X.-A. \& Yu, S. Diastereoselective and Stereodivergent Synthesis of 2-Cinnamylpyrrolines Enabled by Photoredox-Catalyzed Iminoalkenylation of Alkenes. Angew. Chem. Int. Ed. 60, 9672-9679 (2021).

44. Sakurai, S., Matsumoto, A., Kano, T. \& Maruoka, K. Cu-Catalyzed Enantioselective Alkylarylation of Vinylarenes Enabled by Chiral Binaphthyl-BOX Hybrid Ligands. J. Am. Chem. Soc. 142, 19017-19022 (2020).

45. Rossler, S. L. et al. Pyridinium Salts as Redox-Active Functional Group Transfer Reagents. Angew. Chem. Int. Ed. 59, 9264-9280 (2020).

46. Crespi, S. \& Fagnoni, M. Generation of Alkyl Radicals: From the Tyranny of Tin to the Photon Democracy. Chem. Rev. 120, 9790-9833 (2020).

47. Oldenziel, O. H., Van Leusen, D. \& Van Leusen, A. M. A general one-step synthesis of nitriles from ketones using tosylmethyl isocyanide. Introduction of a one-carbon unit. J. Org. Chem. 42, 3114-3118 (1977).

48. Liu, J. et al. Visible-light-induced triple catalysis for a ring-opening cyanation of cyclopropyl ketones. Chem. Commun. 56, 11508-11511 (2020).

49. Wagaw, S., Yang, B. H. \& Buchwald, S. L. A Palladium-Catalyzed Strategy for the Preparation of Indoles: A Novel Entry into the Fischer Indole Synthesis. J. Am. Chem. Soc. 120, 6621-6622 (1998).

50. Yao, X. et al. Palladium-Catalyzed Cascade Reactions of $\delta$-Ketonitriles with Arylboronic Acids: Synthesis of Pyridines. ACS Comb. Sci. 22, 114-119 (2020).

51. Liu, J. et al. Visible-Light-Mediated Decarboxylation/Oxidative Amidation of $\alpha$-Keto Acids with Amines under Mild Reaction Conditions Using $\mathrm{O}_{2}$. Angew. Chem. Int. Ed. 53, 502-506 (2014).

52. Kawamoto, T., Matsubara, H., Fukuyama, T. \& Ryu, I. A Theoretical Study on Radical-based Aminocarbonylation of Aryl lodides. Chem. Lett. 47, 1169-1171 (2018).

53. Ganley, J. M., Murray, P. R. D. \& Knowles, R. R. Photocatalytic Generation of Aminium Radical Cations 
for C-N Bond Formation. ACS Catal., 11712-11738 (2020).

54. Parsaee, F. et al. Radical philicity and its role in selective organic transformations. Nat. Rev. Chem. 5, 486-499 (2021).

55. L, B. et al. Switchable radical carbonylation by polarity-regulation. Preprint at ChemRxiv

\section{ACKNOWLEDGMENTS}

Funding: We are grateful to the financial support from the National Natural Science Foundation of China (21971081, 22171099, 91856119, 21772053, 21820102003, 91956201, and 21773025), the Program of Introducing Talents of Discipline to Universities of China (111 Program, B17019), and the Fundamental Research Funds for the Central Universities (WHU 2042021KF1020). The numerical calculations in this paper have been done on the supercomputing system in the Supercomputing Center of Wuhan University. Author contributions: W.-J.X. and J.-R.C. conceived the project and supervised the research. B.L. are responsible for the implementation of the experimental work. X.Q. is responsible for implementation of the computational studies and the writing of corresponding mechanistic discussions. W.-J.X. and J.-R.C. co-write the manuscript with revisions provided by the other authors. All authors discussed the results and commented on the manuscript. Competing interests: The authors declare no conflicts of interest. Data and materials availability: All data is available in the main text or the supplementary materials. 